\title{
Spectroscopy Results from HERA
}

François Corriveau, IPP/McGill

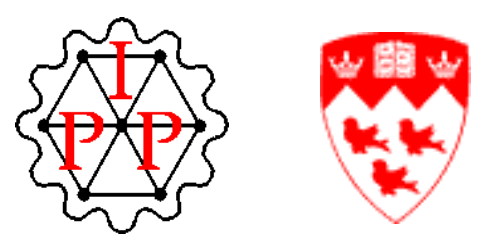

on behalf of the $\mathrm{H} 1$ and ZEUS Collaborations

PhiPsi08 at Laboratori Nazionali di Frascati

10 April 2008

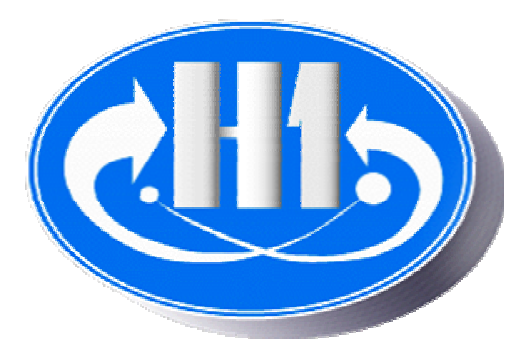

- H1 and ZEUS at HERA

- Hadron production

- Tetraquark States?

- Pentaquark States?

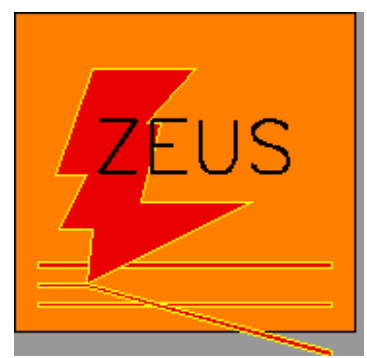

- Outlook 


\section{$\mathrm{e}^{ \pm} \mathrm{p}-$ Kinematics at HERA}

27.6 GeV electrons/positrons on 920(820) GeV protons

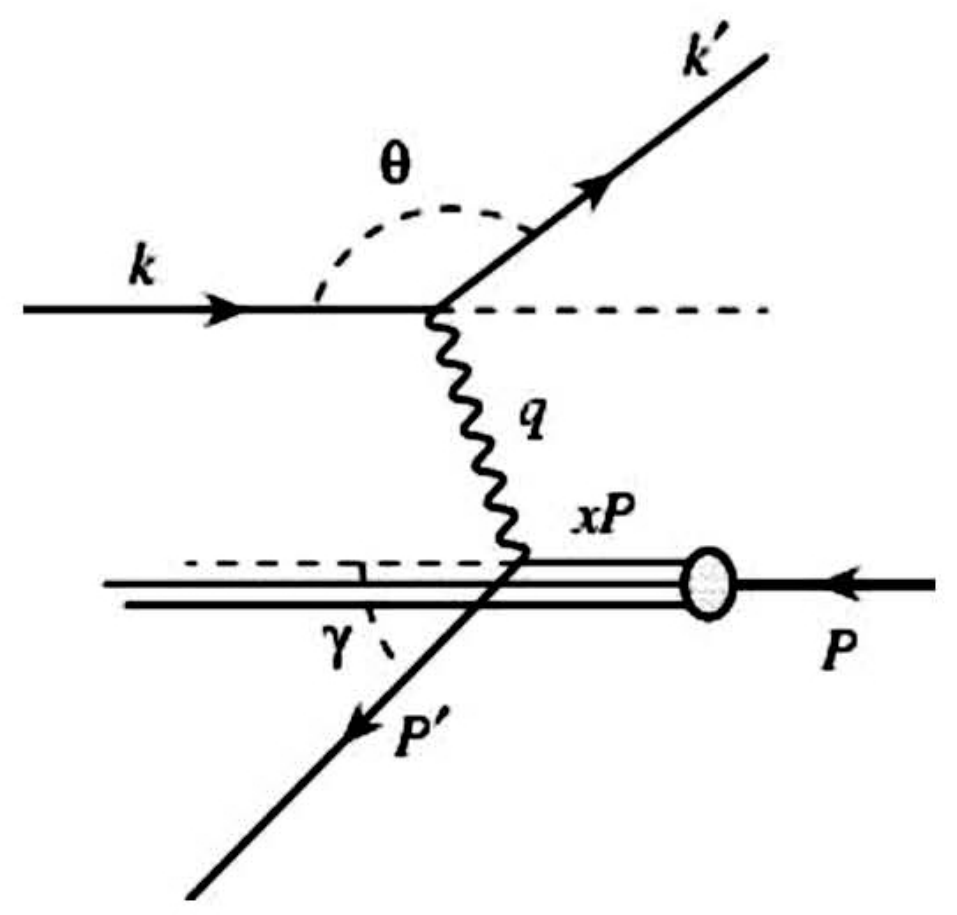

\section{Deep Inelastic Scattering (DIS):}

Neutral current (NC) via $\gamma / Z^{0}$ exchange Charged current (CC) via $\mathrm{W}^{ \pm}$exchange Photoproduction: $\mathbf{Q}^{2} \approx 0$

$$
\begin{aligned}
& Q^{2}=-q^{2}=-\left(k-k^{\prime}\right)^{2} \\
& x=\frac{Q^{2}}{2 p \cdot q} \quad y=\frac{p \cdot q}{p \cdot k} \\
& s=(p+k)^{2} \quad Q^{2}=x \cdot y \cdot s
\end{aligned}
$$

$\mathrm{Q}^{2}=$ exchanged momentum (squared)

$\mathrm{x}=$ Bjorken scaling variable

y = inelasticity scaling variable

$\sqrt{ } \mathrm{s}=$ center of mass energy $(\sim 320 \mathrm{GeV})$

$\mathrm{W}=$ photon-proton center of mass energy 


\section{HERA Luminosities}

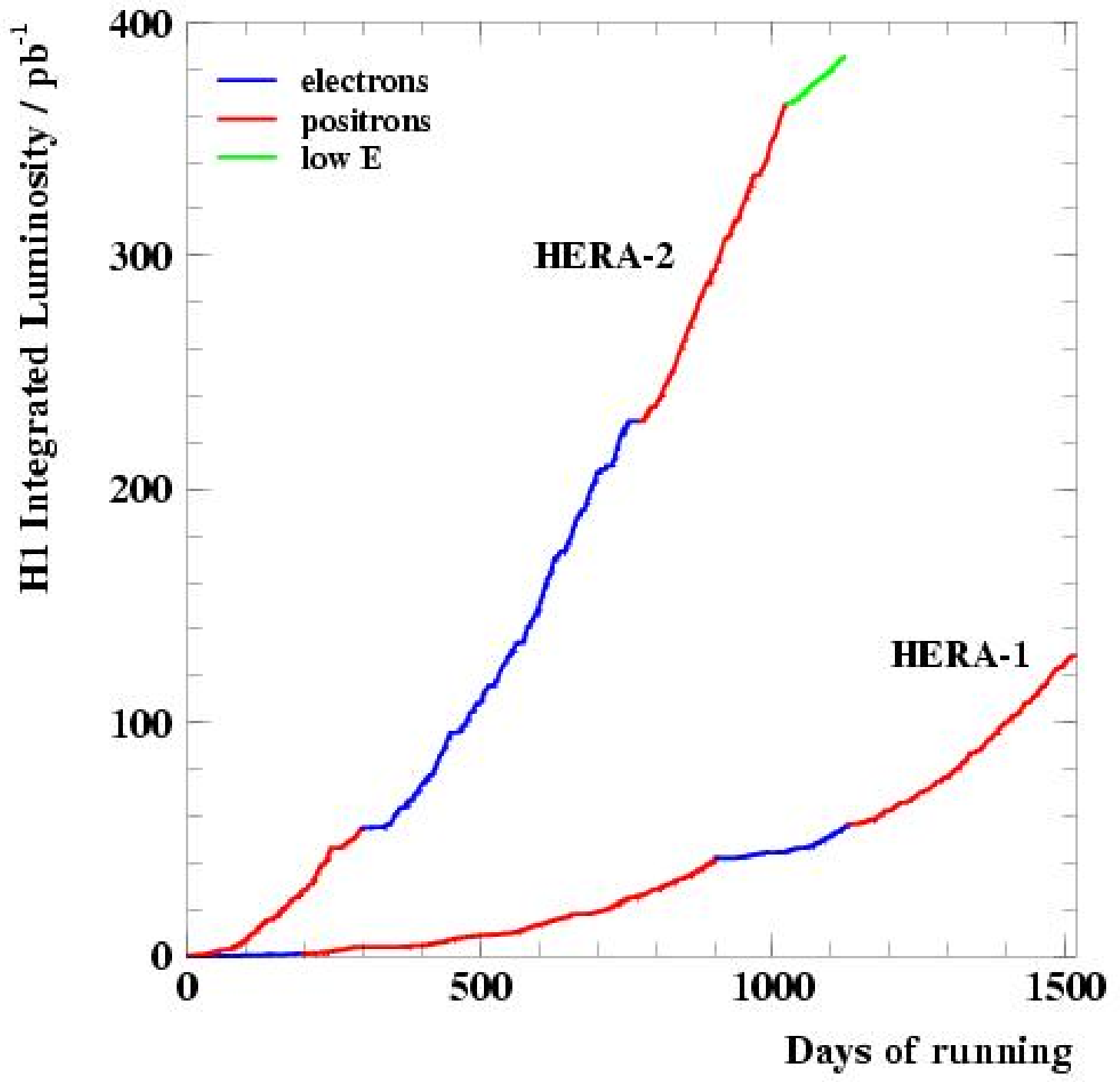

\section{HERA I: unpolarised $\mathbf{e}^{ \pm}$beams \\ HERA II: polarised $\mathrm{e}^{ \pm}$beams}

Gated luminosities

\begin{tabular}{|c|c|c|}
\hline e$^{ \pm} \mathbf{p}$ & H1 & ZEUS \\
\hline HERA I & $128 \mathrm{pb}^{-1}$ & $143 \mathrm{pb}^{-1}$ \\
\hline HERA II & $385 \mathrm{pb}^{-1}$ & $407 \mathrm{pb}^{-1}$ \\
\hline
\end{tabular}

$\sim 0.5 \mathrm{fb}^{-1}$ per experiment 


\section{Hadron Production at HERA}

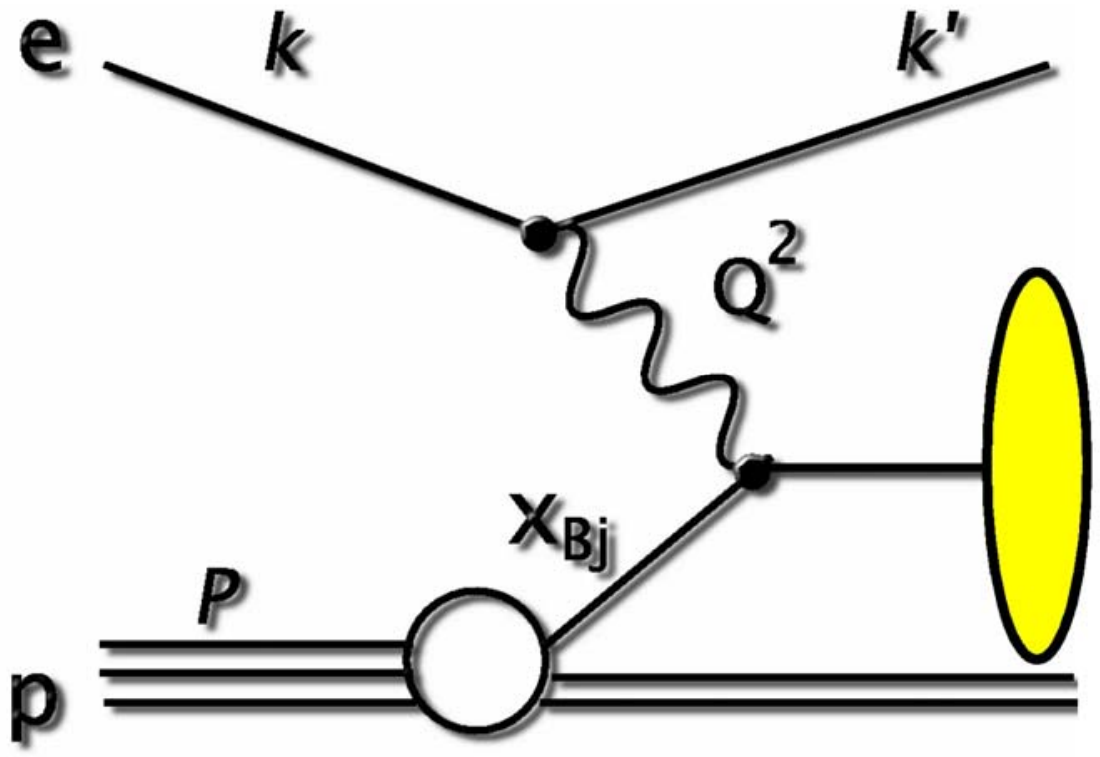

Hadronisation processes leading to hadronic final states (non-perturbative QCD)

Heavy quark production however dominated by photon-gluon processes (perturbative QCD)

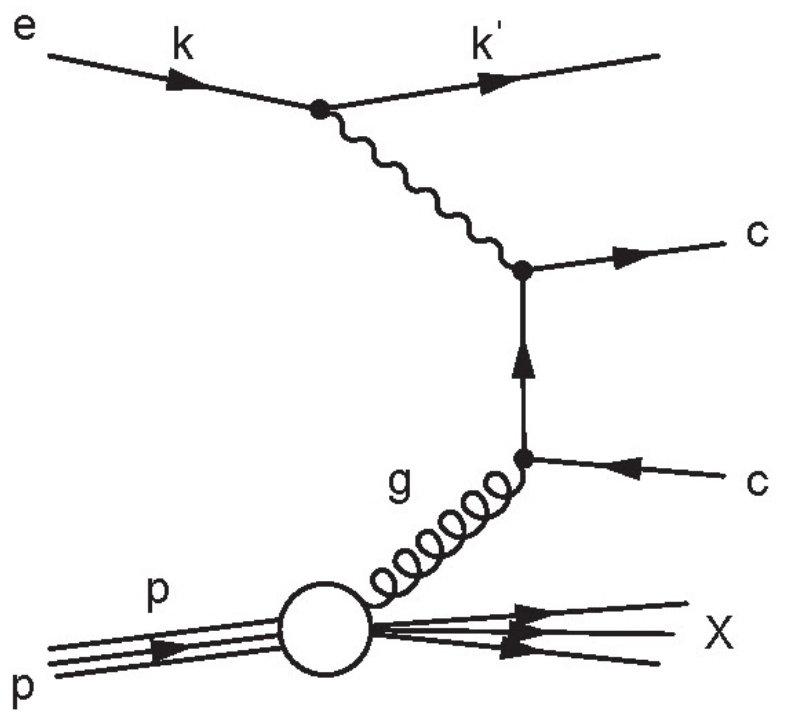




\section{Strangeness}

Production:

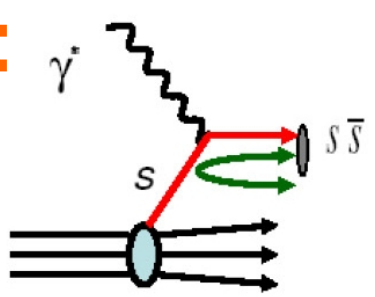

a) Hard scattering of $s$ sea quark

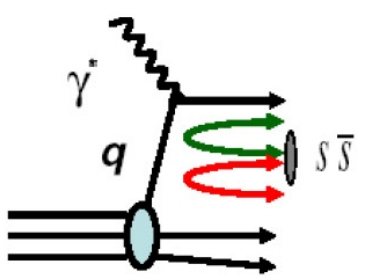

c) Parton pure fragmentation

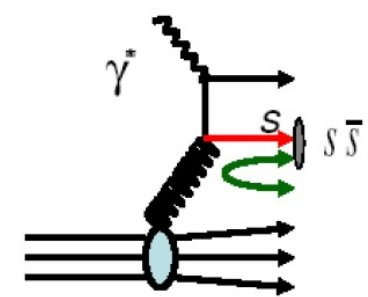

b) Boson-gluon fusion (BGF)

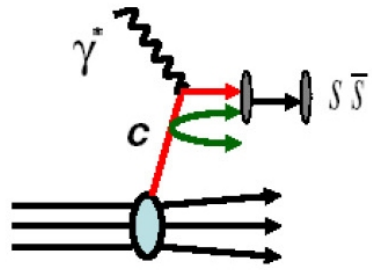

d) Heavy quark decay
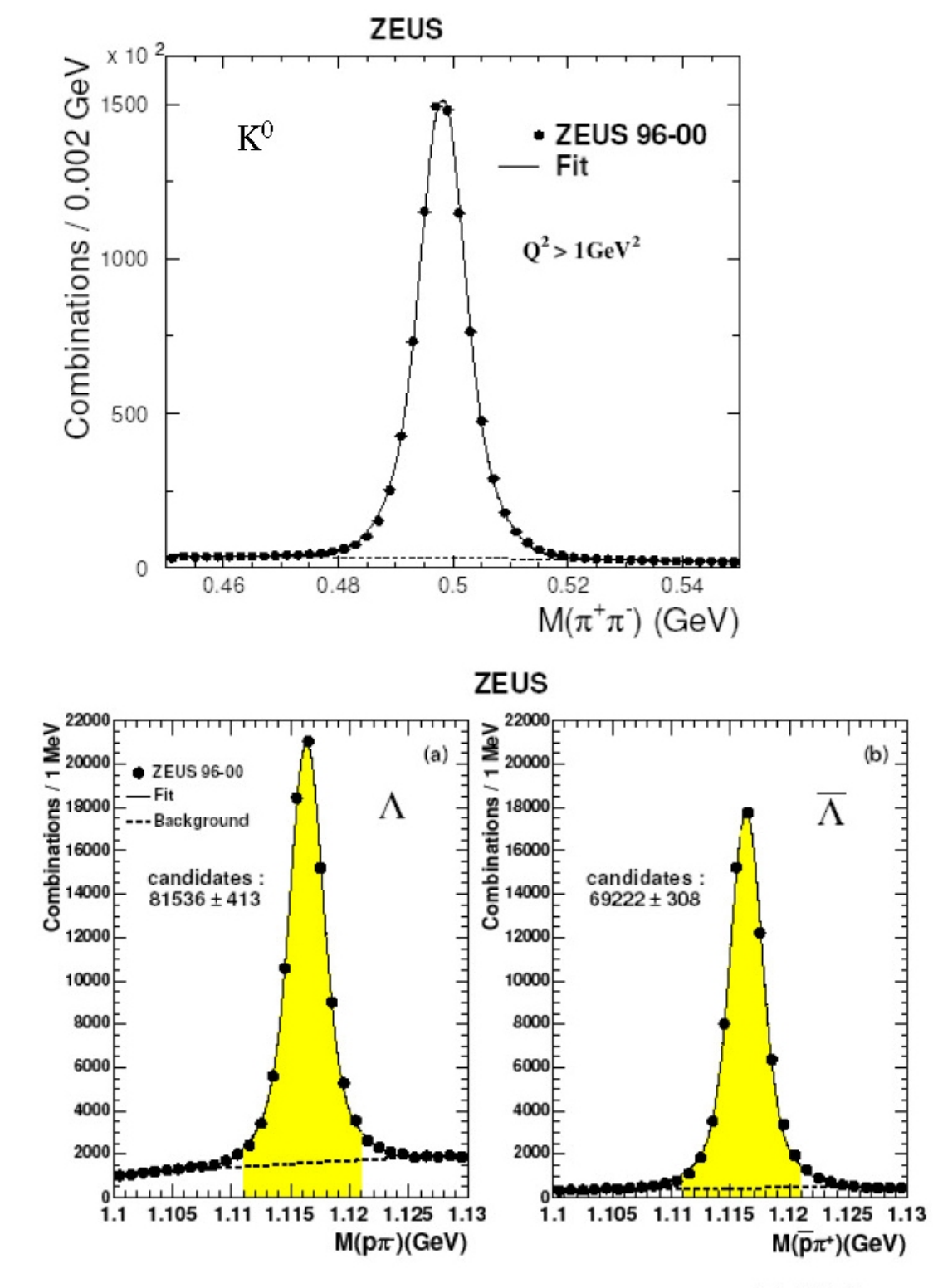

\section{Observation of $\mathrm{K}_{\mathrm{s}}{ }^{\prime}$ 's and $\Lambda$ 's:}

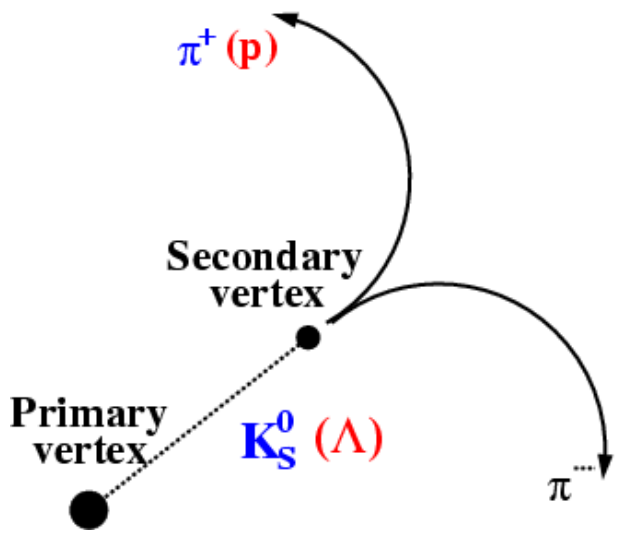




\section{Strangeness Results}

Baryon to meson ratio: $(\Lambda+\bar{\Lambda}) / \mathrm{K}_{\mathrm{s}}^{0}$
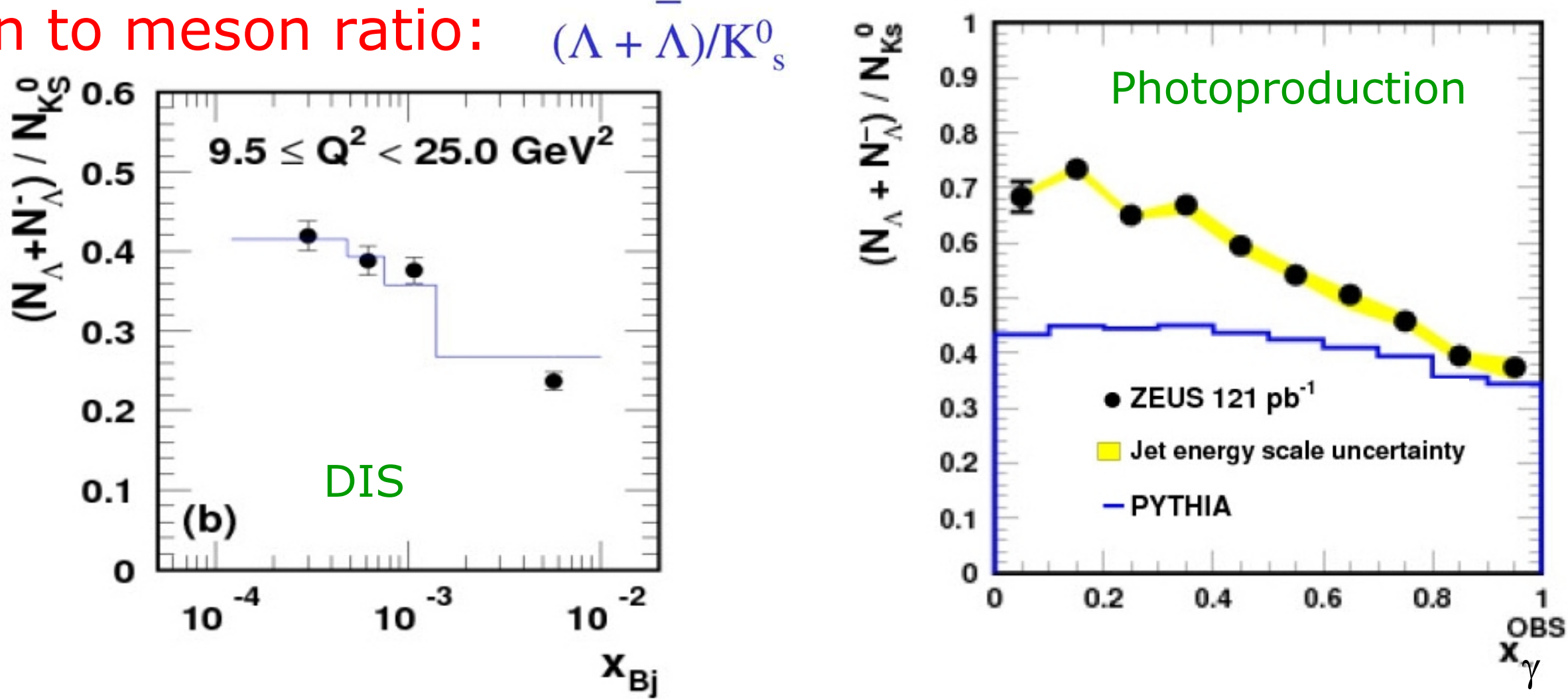

fairly well described by ARIADNE MC (strangeness suppression factor $\lambda_{s}=0.3$ )

new production mechanism needed at low $x_{\gamma}{ }^{\text {OBS }}$

full sets of cross sections vs event/particle variables

Other results:

(similar ones from $\mathrm{H} 1$ ) baryon to meson ratio similar to $\mathrm{e}^{+} \mathrm{e}^{-}$measurements no baryon-antibaryon asymmetry observed no evidence for $\wedge$ transverse polarisation 


\section{Charm Production}

\begin{tabular}{|c|}
\hline$D^{0} \rightarrow K^{-} \pi^{+}$ \\
\hline$D^{*+} \rightarrow D^{0} \pi_{s}^{+}$ \\
\hline$D^{+} \rightarrow K^{-} \pi^{+} \pi^{+}$ \\
\hline$D_{s}^{+} \rightarrow \phi \pi^{+} \rightarrow K^{+} K^{-} \pi^{+}$ \\
\hline$\Lambda_{c}^{+} \rightarrow K^{-} p \pi^{+}$ \\
\hline
\end{tabular}

- charm fragmentation ratios

- charm hadronisation fractions

- .. numerous other QCD results

(cross sections vs QCD NLO, $\mathrm{F}_{2}{ }^{c c}, .$. ) 


\section{$D^{0}$ and D* Production}

ZEUS
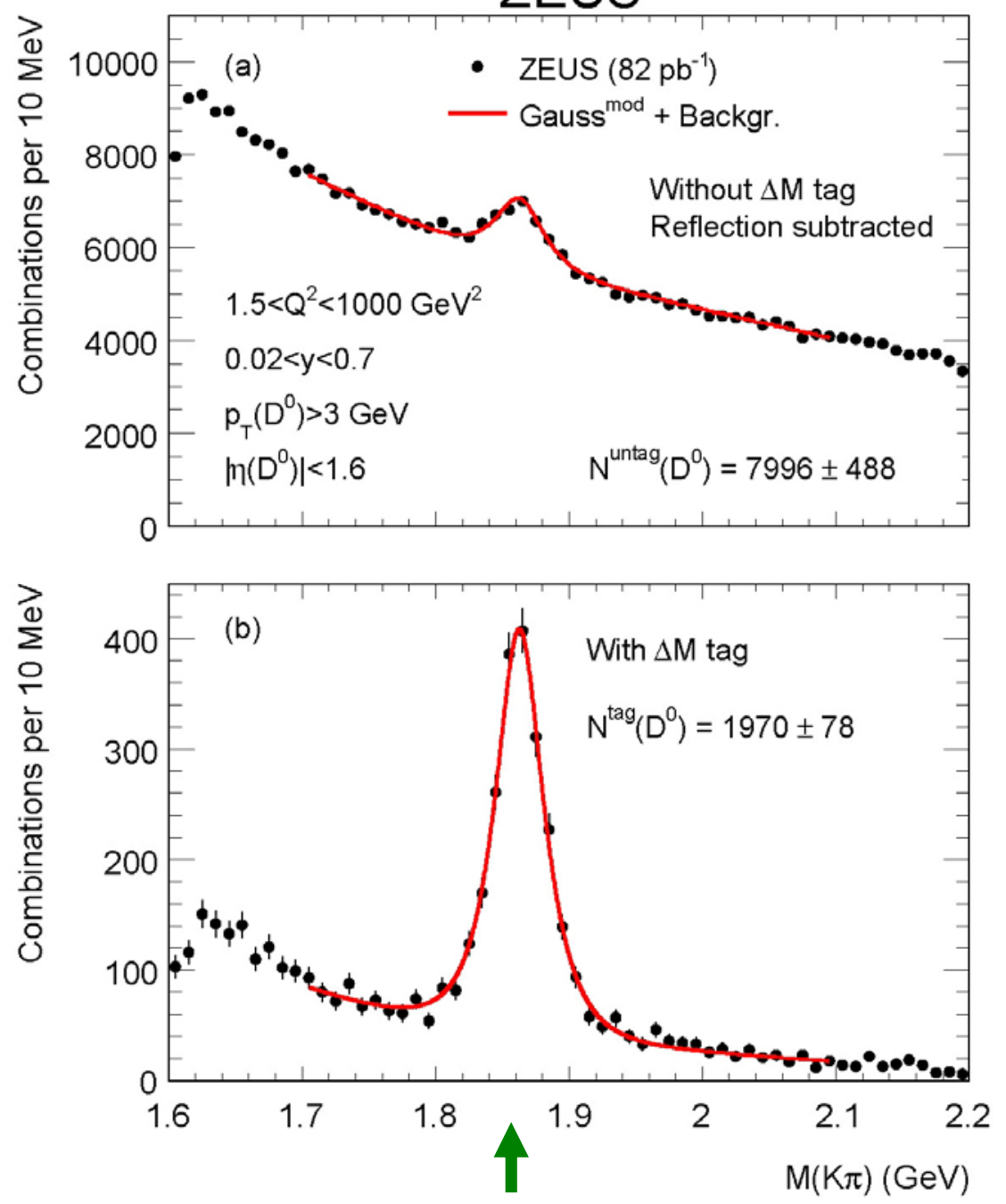

$\Delta \mathrm{M}$ tag from $\mathrm{D}^{*+} \rightarrow \mathrm{D}^{0} \pi_{\mathrm{s}}{ }^{+}$events

\section{ZEUS}

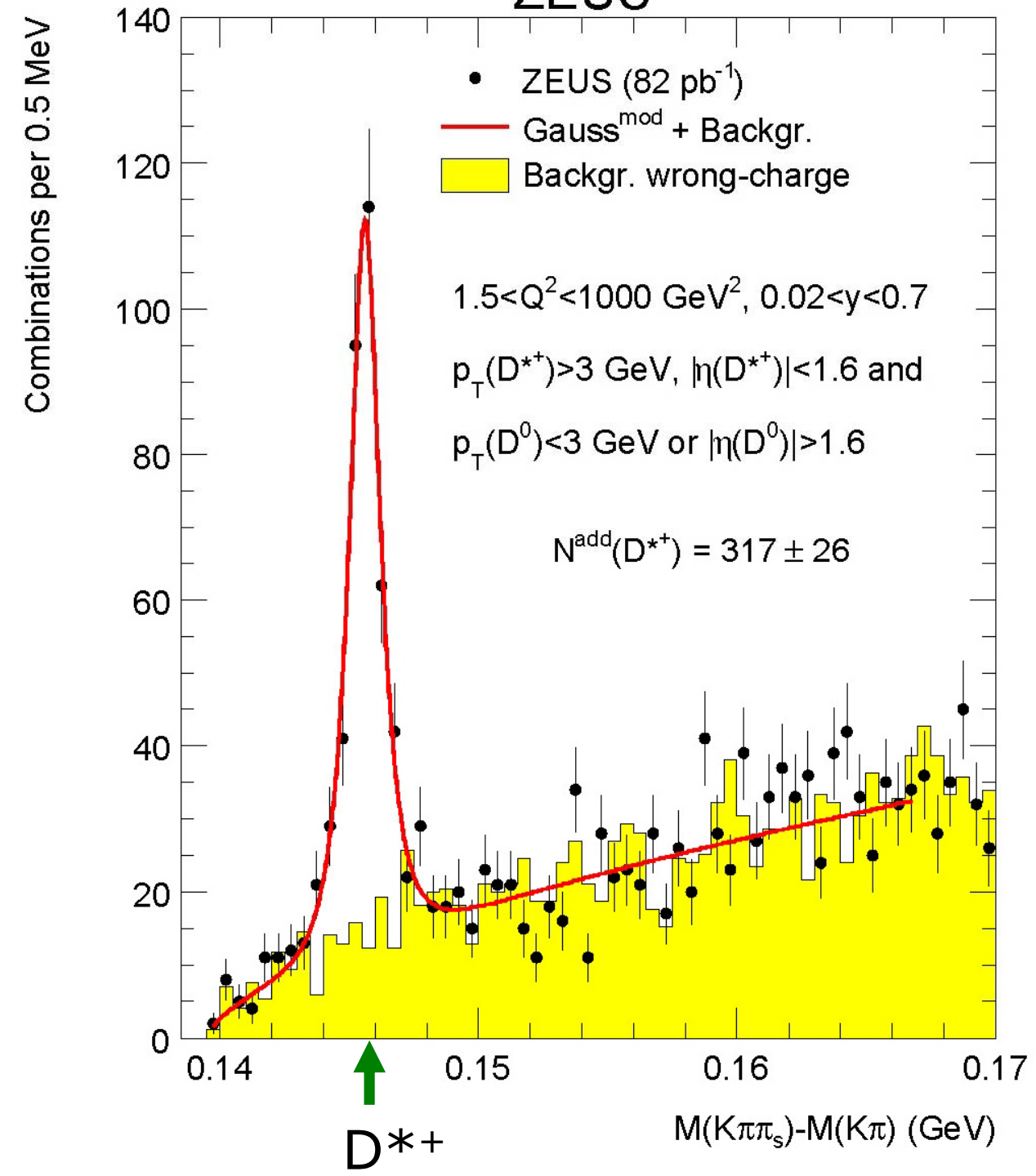




\section{$\mathrm{D}^{+}$and $\mathrm{D}_{\mathrm{s}}^{+}$Production}

\section{ZEUS}

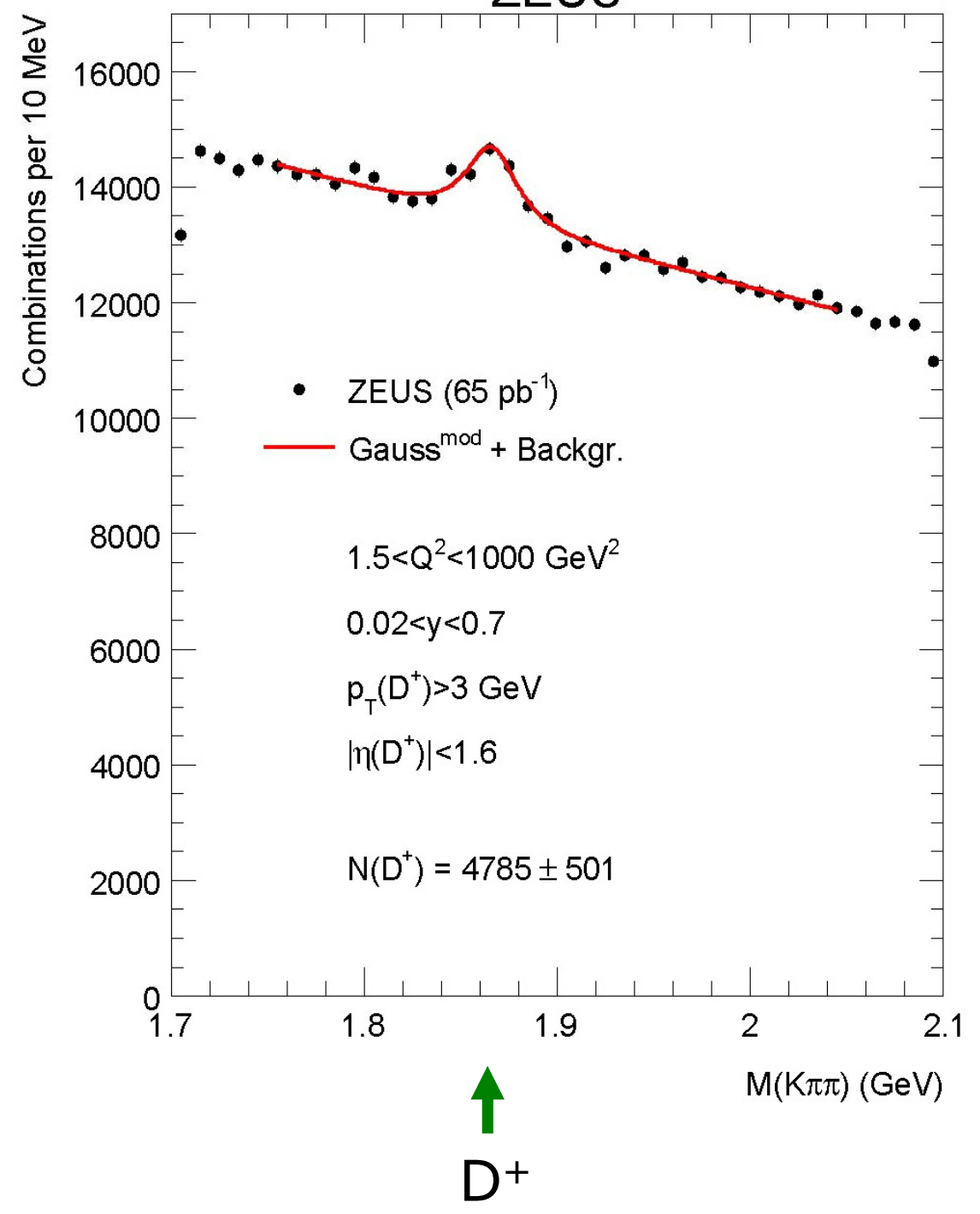

\section{ZEUS}
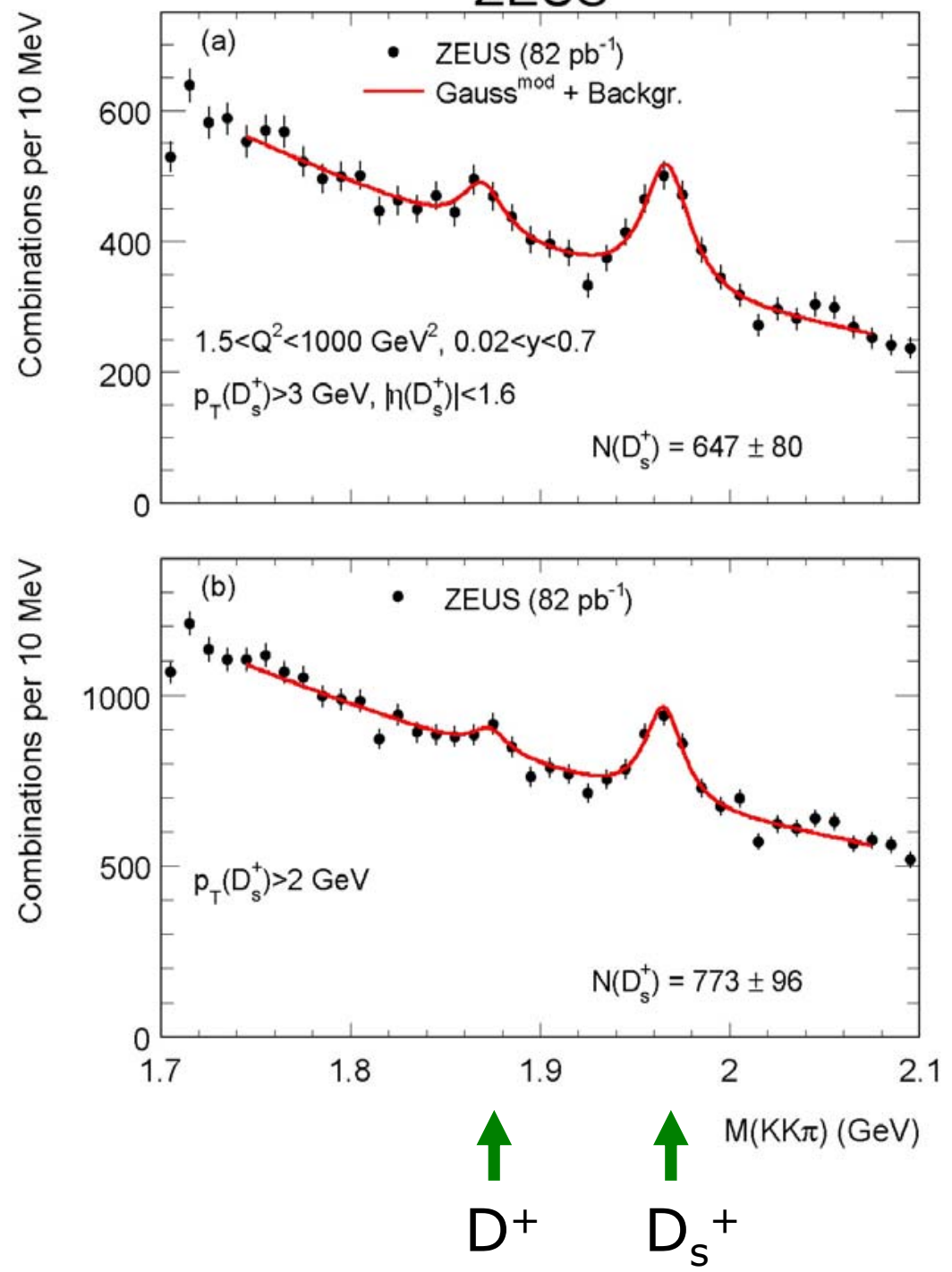


\section{Charm Fragmentation}

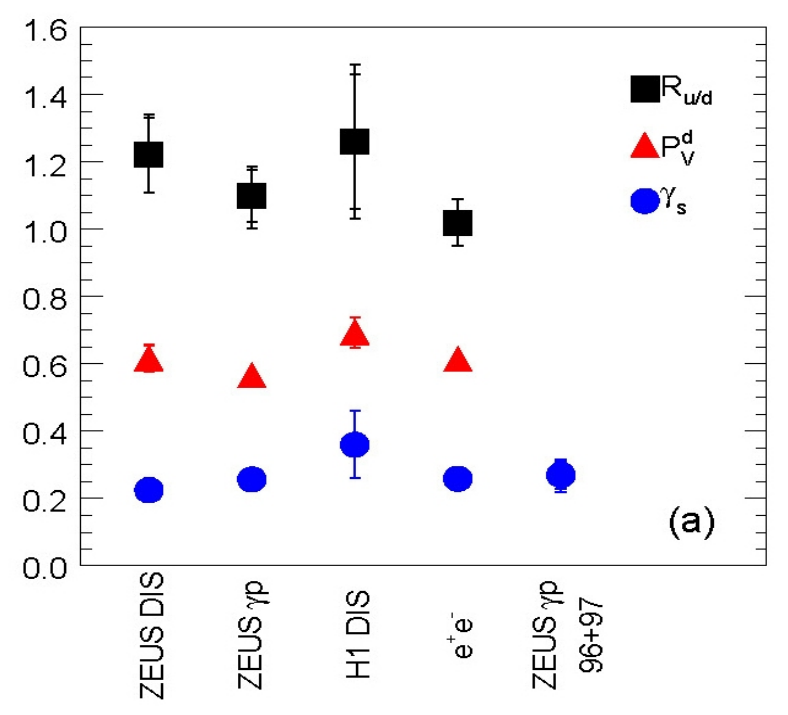

\section{Ratios:}

- neutral to charged D mesons $\rightarrow \mathrm{u}$ and $\mathrm{d}$ produced equally in charm fragmentation

- fraction charged D's in vector state < naïve 3/4

- strangeness suppression factor

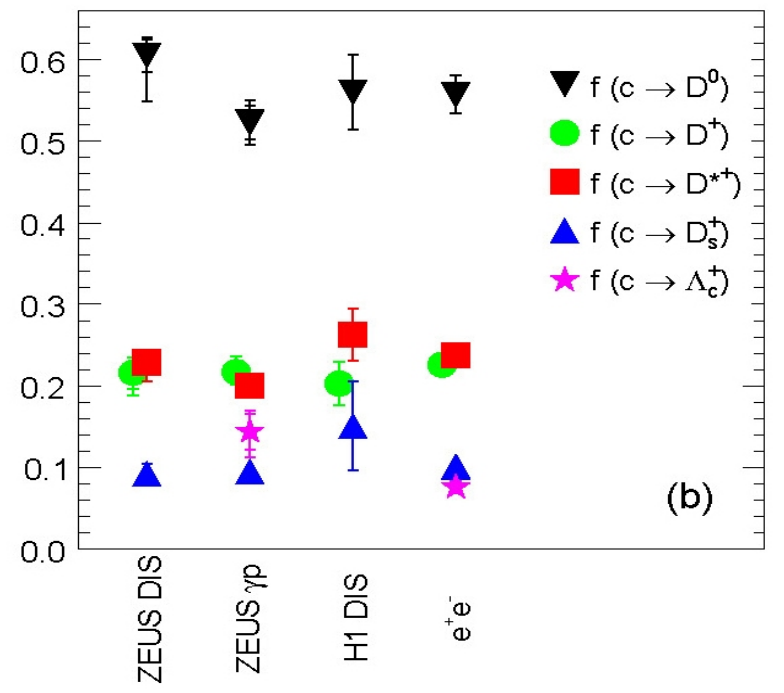

\section{Fractions:}

- generally consistent with expectations

- agreements ZEUS - $\mathrm{H} 1-\mathrm{e}^{+} \mathrm{e}^{-}$

- fragmentation independent of the hard subprocesses 


\section{Gluon and Quark States}

The best known hadrons are well explained by the Standard Model

Particles made only from gluons, or from more than 3 quarks are not excluded

Since gluons carry both color and anti-color, 2 or 3 may form color singlet "glueballs"

\section{Lattice QCD calculations predict:}

lightest glueball $\mathrm{JPC}^{\mathrm{PC}}=\mathrm{0}^{++}$in mass range $1450-1750 \mathrm{MeV}$ next: $J^{P C}=2^{++}$in mass range $2300-2600 \mathrm{MeV}$

$\mathrm{K}_{\mathrm{s}}{ }^{0} \mathrm{~K}_{\mathrm{s}}{ }^{0}$ bound states: $\mathrm{JC}^{\mathrm{PC}}=0^{++}$(scalar), $2^{++}$(tensor), .. hence may couple to glueballs 


\section{$\mathrm{K}_{\mathrm{s}}{ }^{0} \mathrm{~K}_{\mathrm{s}}{ }^{0}$ Resonant States}

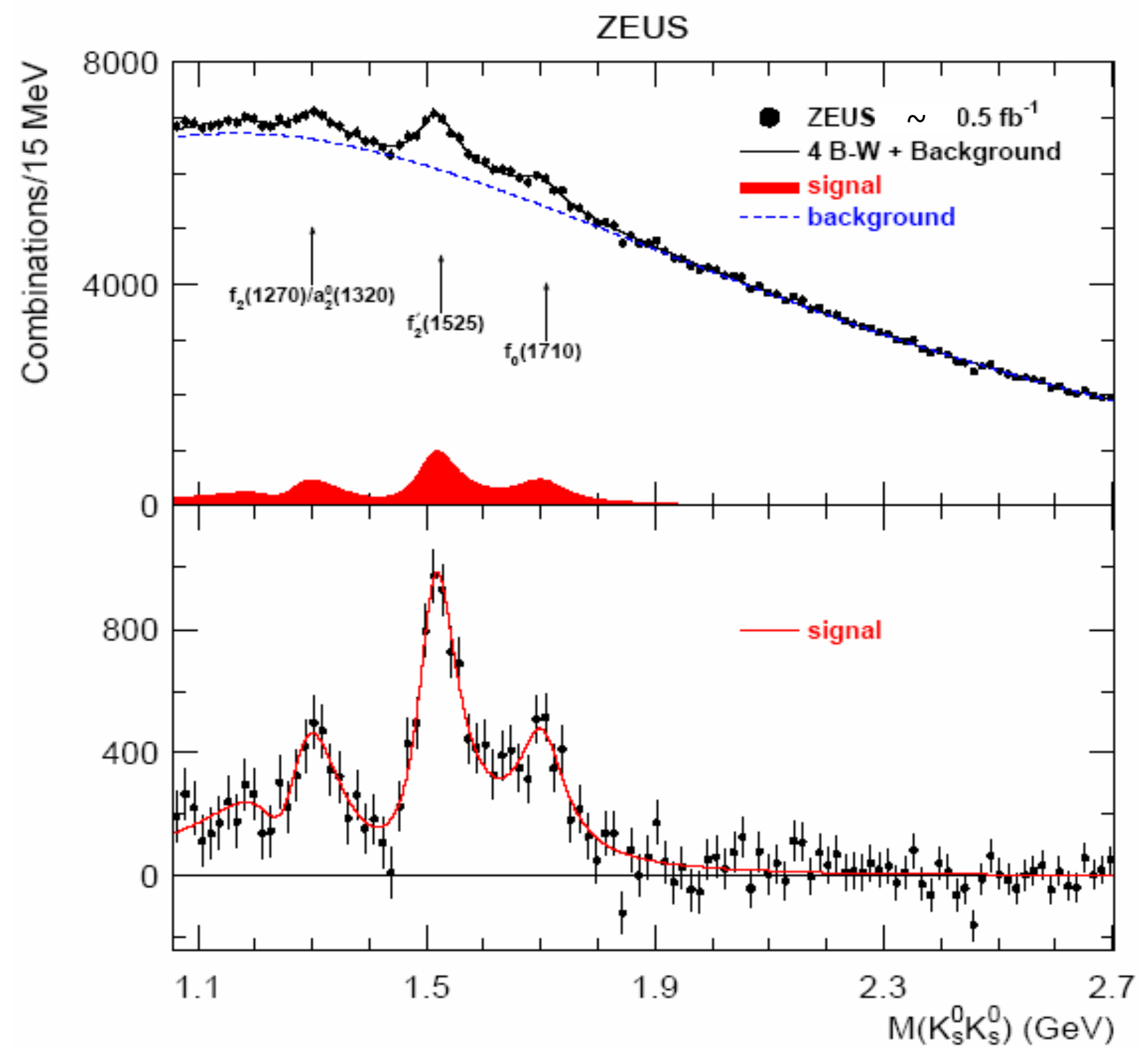

Bret-Wigner functions, with interference terms included

states $f_{2}^{\prime}(1525)$ and $\mathrm{f}_{0}(1710)$ clearly seen

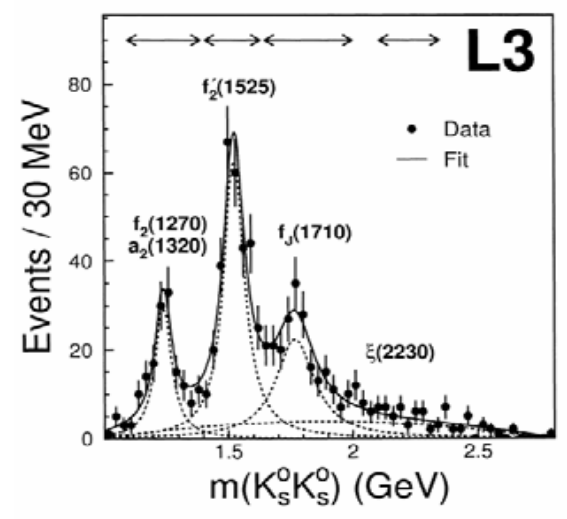




\section{$\mathrm{K}_{\mathrm{s}}{ }^{0} \mathrm{~K}_{\mathrm{s}}{ }^{0}$ Resonance Results}

State $\mathrm{f}_{0}(1710)$ : observed at $5 \sigma$ significance consistent with $\mathrm{JPC}^{\mathrm{PC}} \mathrm{O}^{++}$ glueball candidate

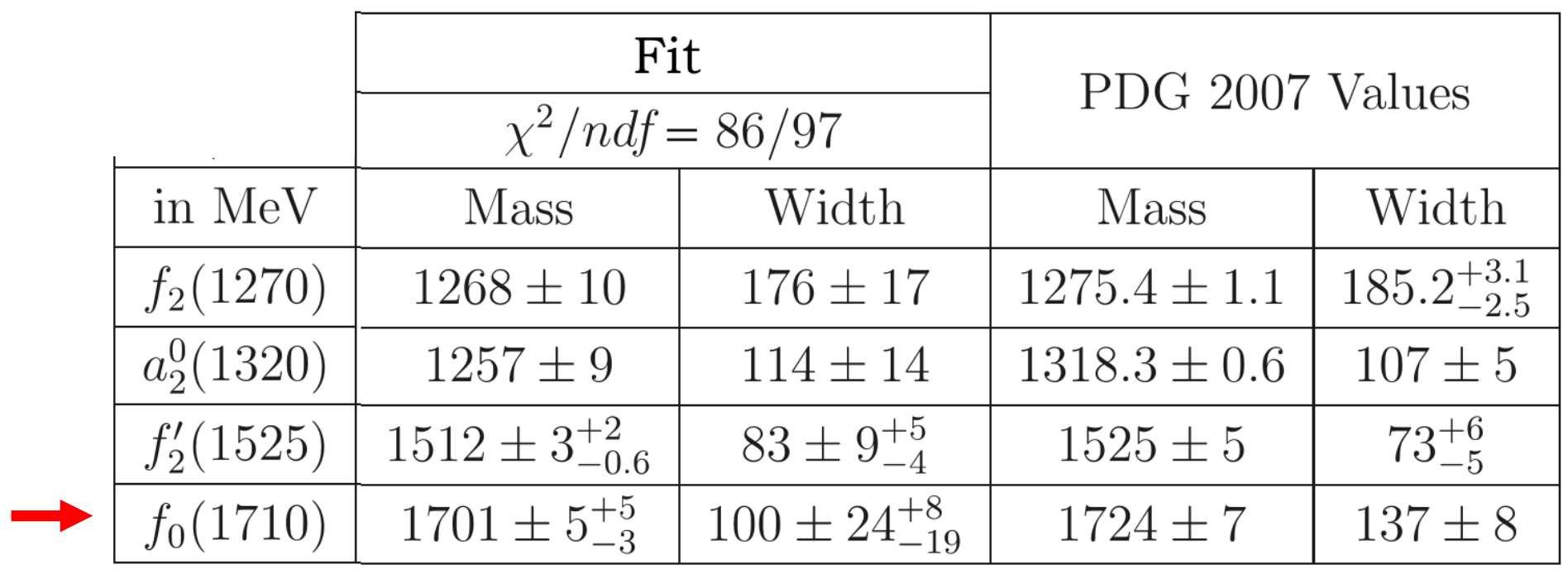

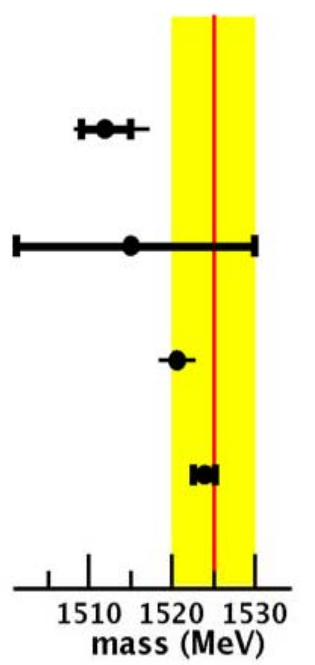

$f_{2}(1525)$ summary

e p ZEUS

Central p p Production

$\mathrm{e}^{+} \mathrm{e}^{-}$experiments

K-meson experiments

$\square$ PDG 2007

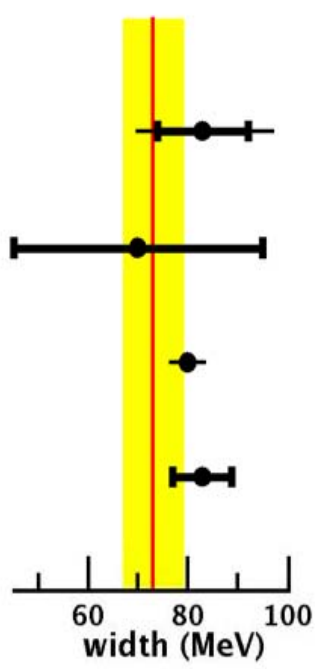

$f_{0}(1710)$ summary

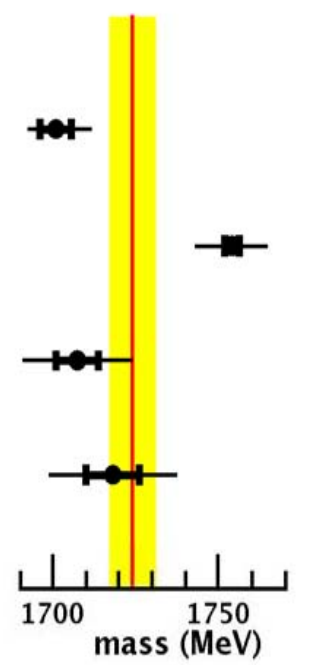

e p ZEUS

$\mathrm{e}^{+} \mathrm{e}^{-}$BES Collab.

$\mathrm{e}^{+} \mathrm{e}^{-}$other Collab.

p p, $\pi$ p experiments

$\square$ PDG 2007

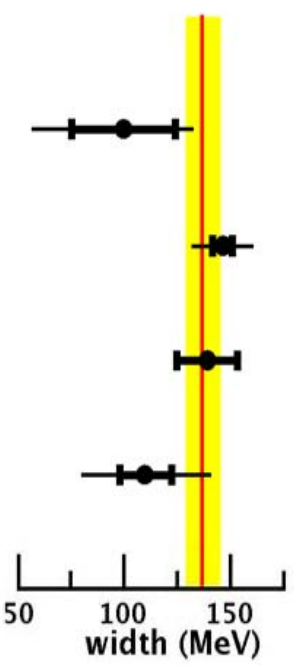




\section{Strangeness States}

Search for: $\quad \Theta^{+} \rightarrow K_{s}^{0} p$ and $\overline{\Theta^{+}} \rightarrow K_{s}^{0} \bar{p}$

\section{ZEUS}

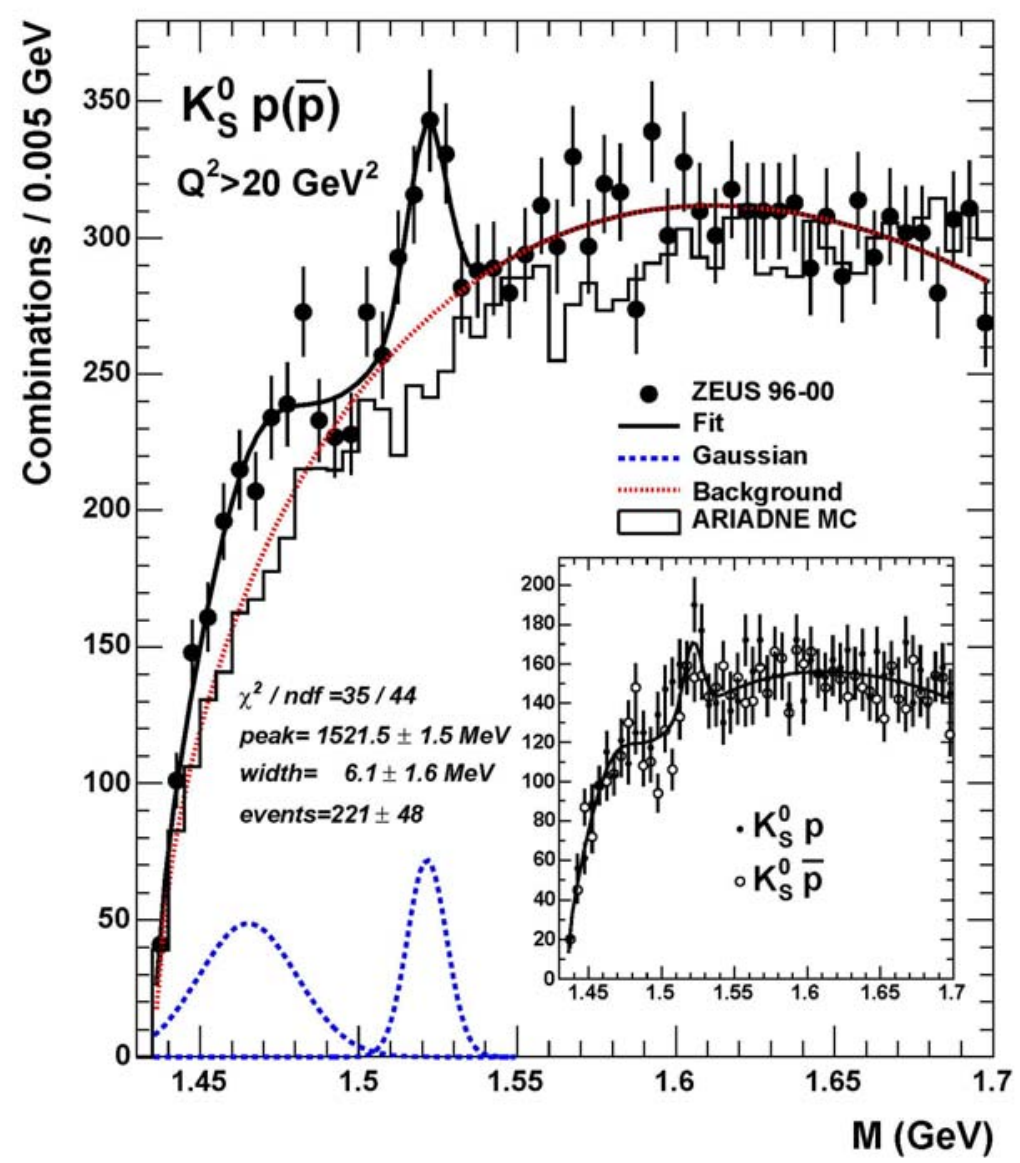

ZEUS has a positive signal at $1522 \mathrm{MeV}$

HERA I data

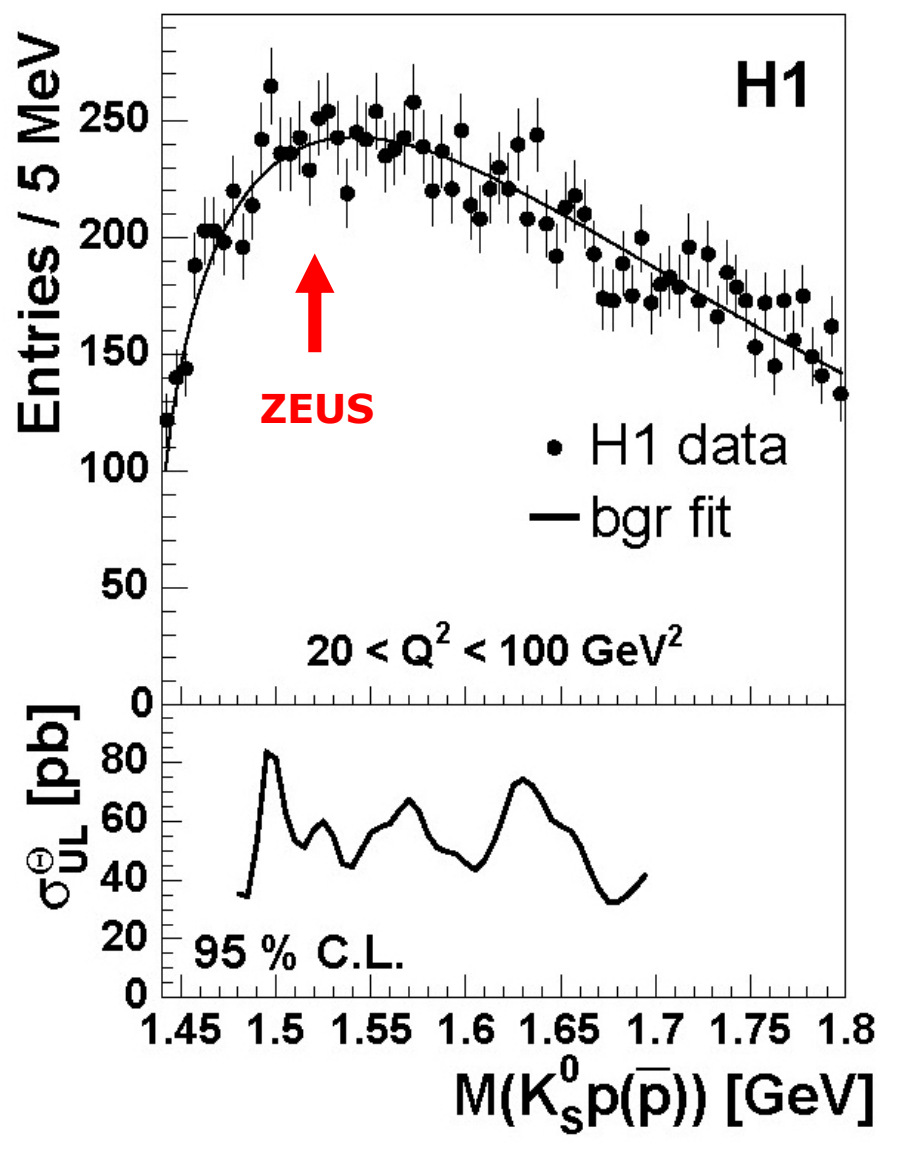

No signal observed in $\mathrm{H} 1$, upper limits set on cross sections. 


\section{Strangeness States}

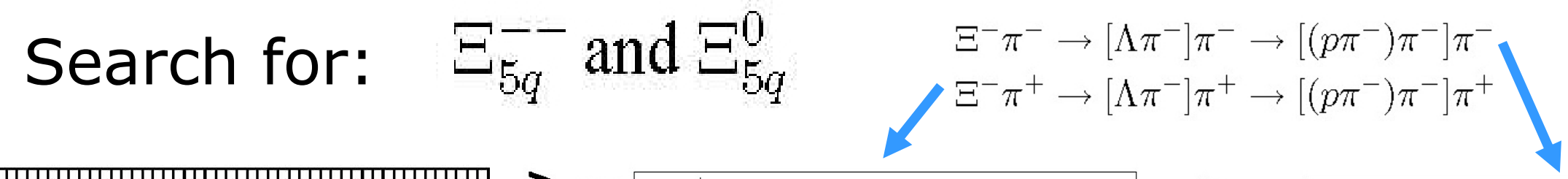
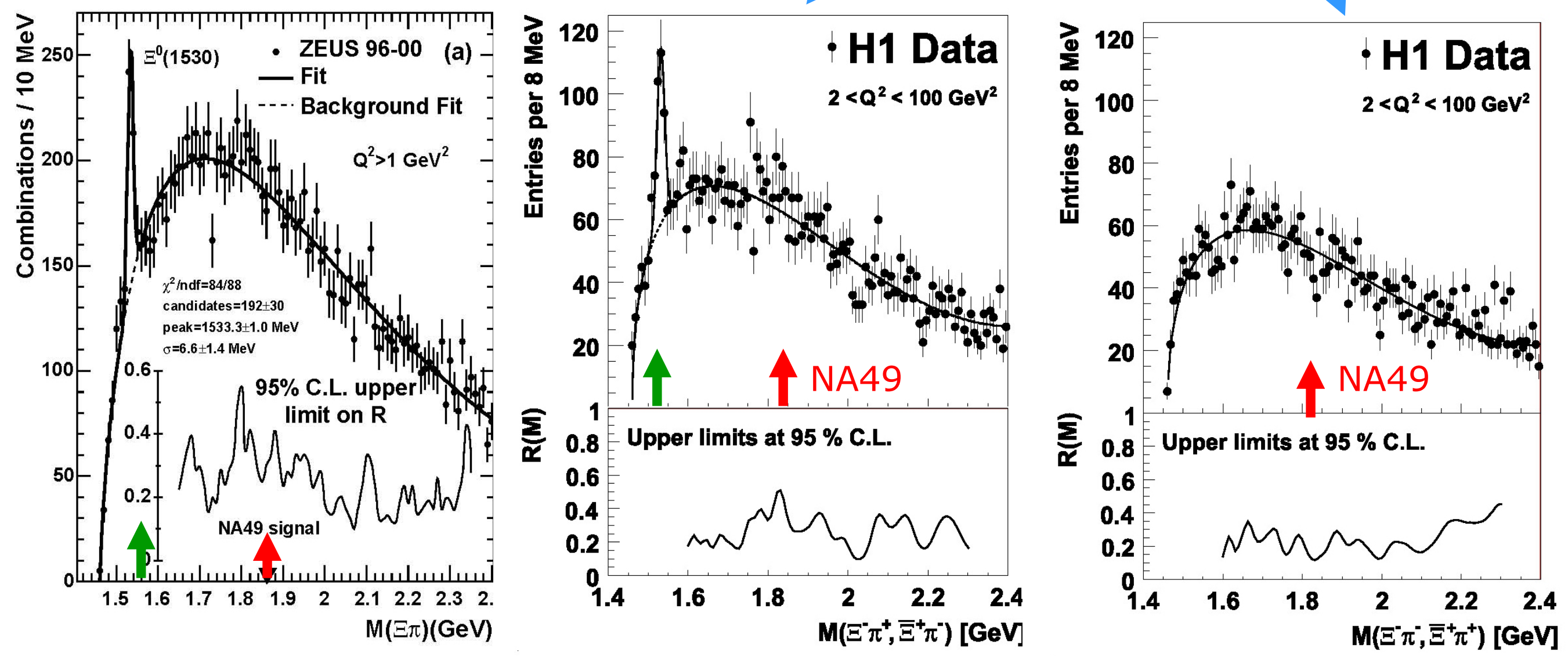

Known resonant state $\equiv(\mathbf{1 5 3 0} \mathbf{M e V})^{0}$. No signal at $1862 \mathrm{MeV}$ (NA49), cross section upper limits set relative to 1530 state. 


\section{D*p Resonance (2004)}

A resonance was observed by $\mathrm{H} 1$ with HERA I data $\left(75 \mathrm{pb}^{-1}\right)$

Invariant mass $=3099 \pm 3 \mathrm{MeV}$

$$
\mathrm{RMS}=12 \pm 3 \mathrm{MeV}
$$
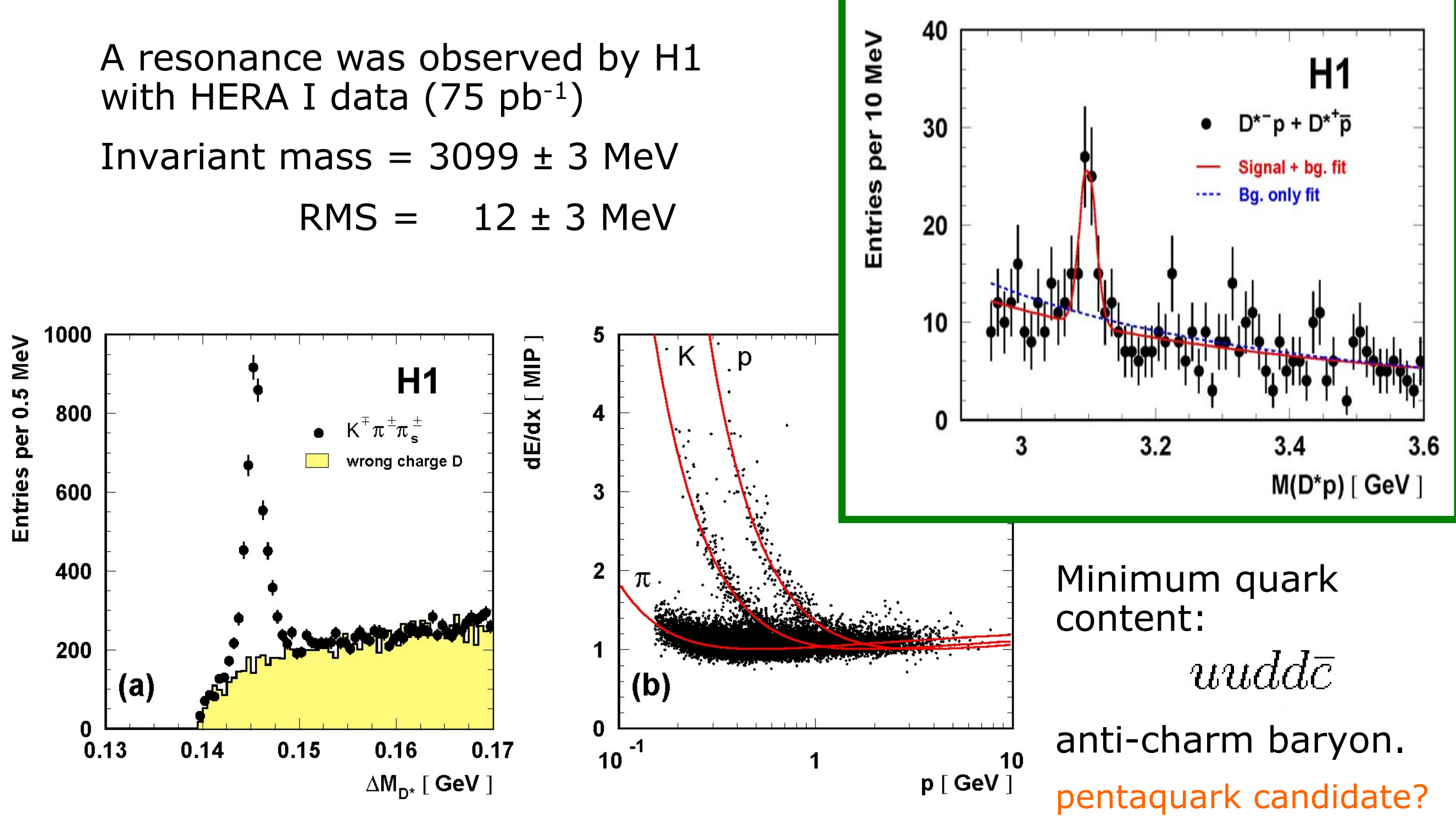


\section{D*p Resonance (2008)}

Excess not observed in other experiments (BaBar, CDF, ZEUS, ALEPH, FOCUS), therefore:

Repeat measurement with HERA II data (348 $\left.\mathrm{pb}^{-1}\right)$ and nearly the same cuts: slightly reduced phase space.
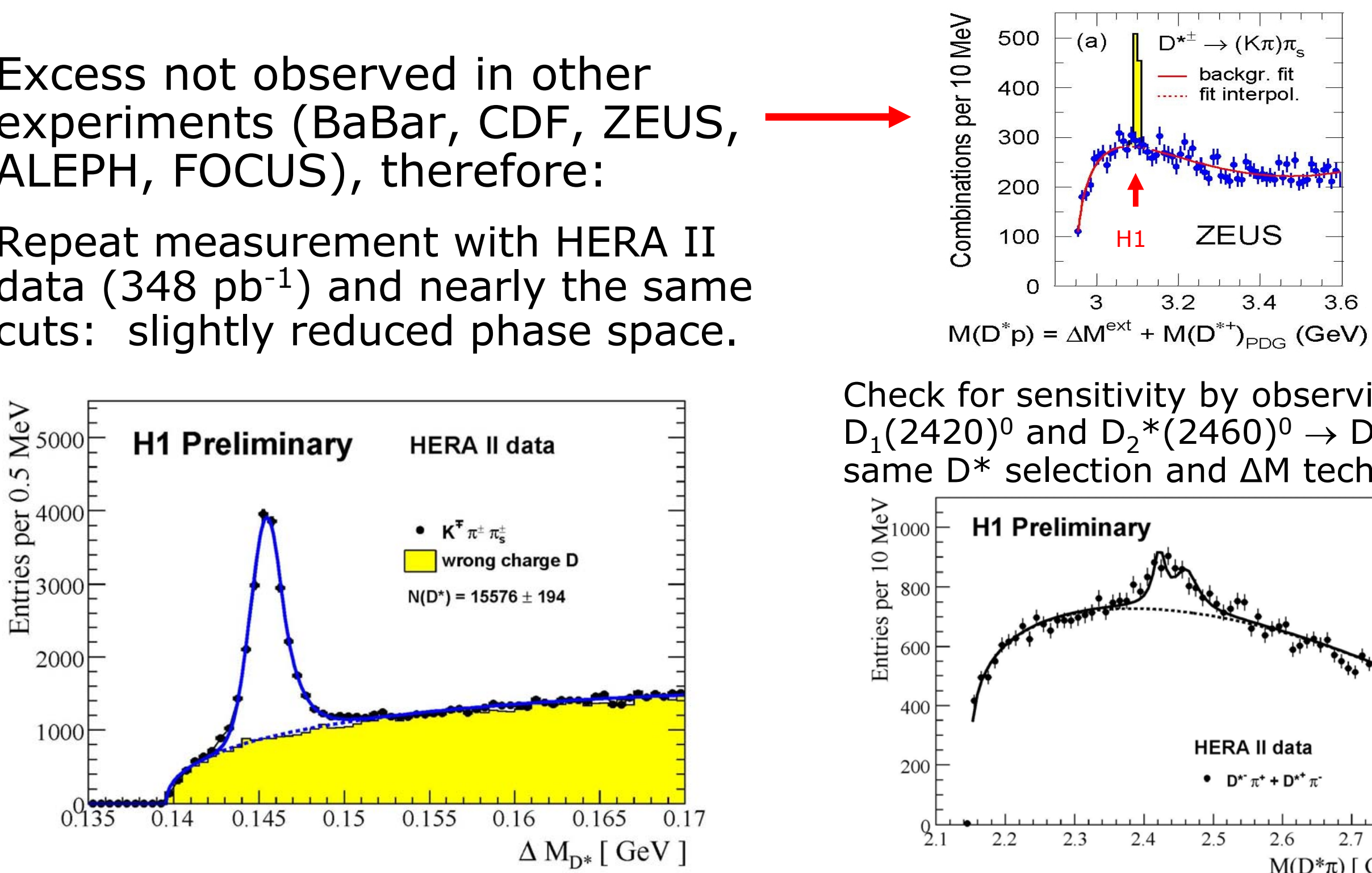

Check for sensitivity by observing $\mathrm{D}_{1}(2420)^{0}$ and $\mathrm{D}_{2} *(2460)^{0} \rightarrow \mathrm{D}^{*} \pi$ : same $D^{*}$ selection and $\Delta M$ technique.

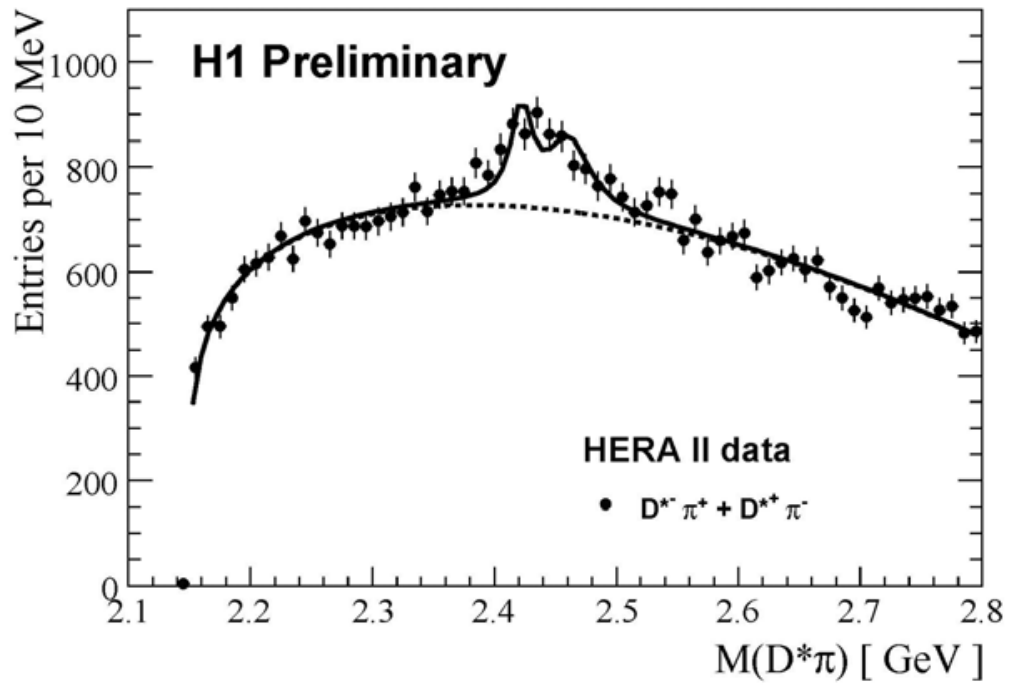




\section{New Analysis}

HERA I data

HERA II phase space high proton momentum

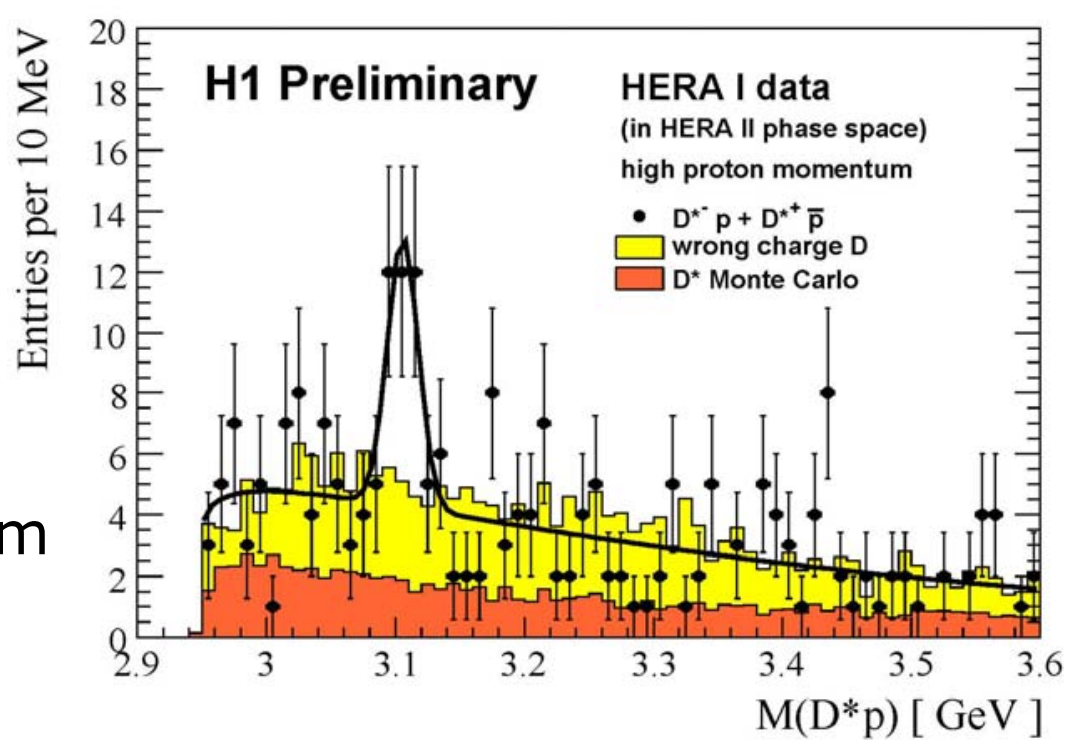

\section{HERA II data}

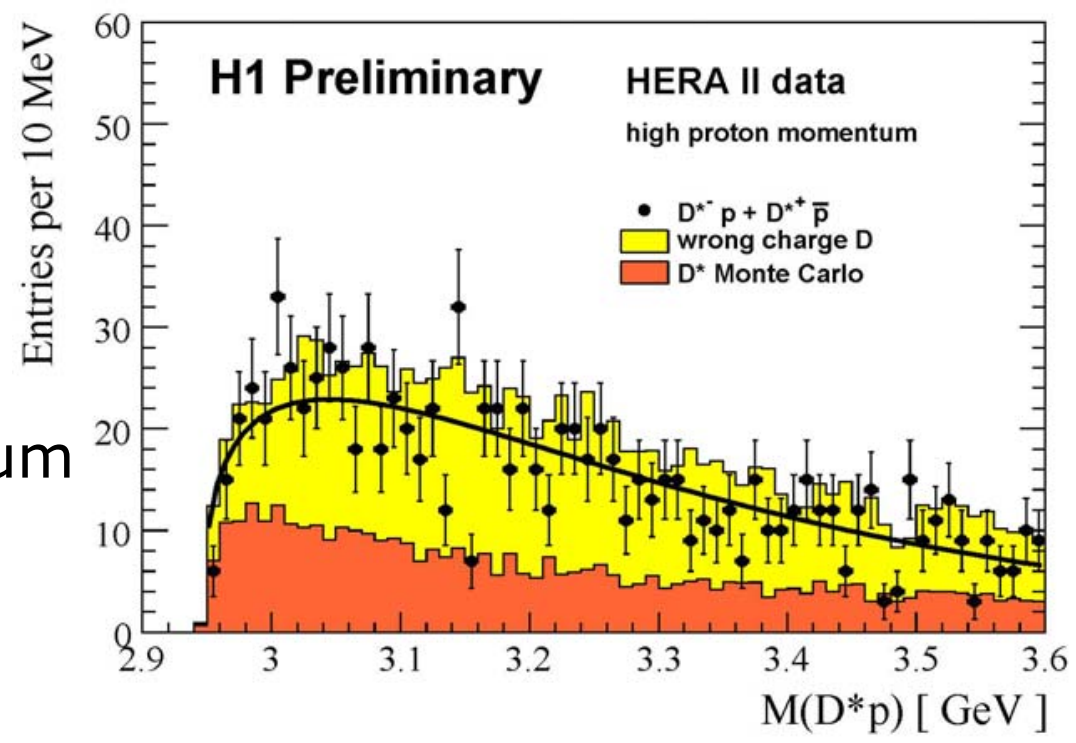

Signal observed in reduced phase space ratio $\mathrm{N}\left(\mathrm{D}^{*} \mathrm{p}\right) / \mathrm{N}\left(\mathrm{D}^{*}\right)$ of $0.81 \pm 0.21 \%$

No peak, $95 \% \mathrm{CL}$ limit of 16.3 events. ratio limit: $0.10 \%$

In both cases: D* Background well described (MC+wrong charge) 


\section{Summary}

- 23 years since conception and after 15 years of operation, HERA stopped on June $30^{\text {th }}, 2007$. An integrated luminosity of $1 \mathrm{fb}^{-1}$ was taken by both experiments $\mathrm{H} 1$ and ZEUS combined.

- ZEUS and H1 have ongoing spectroscopy programs, from light quarks to beauty and for multi-parton resonant state searches.

- HERA II statistics are now being exploited to understand the properties of the investigated states.

- No positive signal observed in the most recent pentaquark searches. 


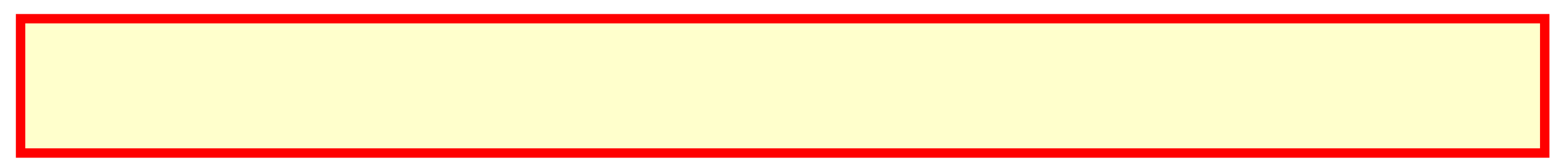

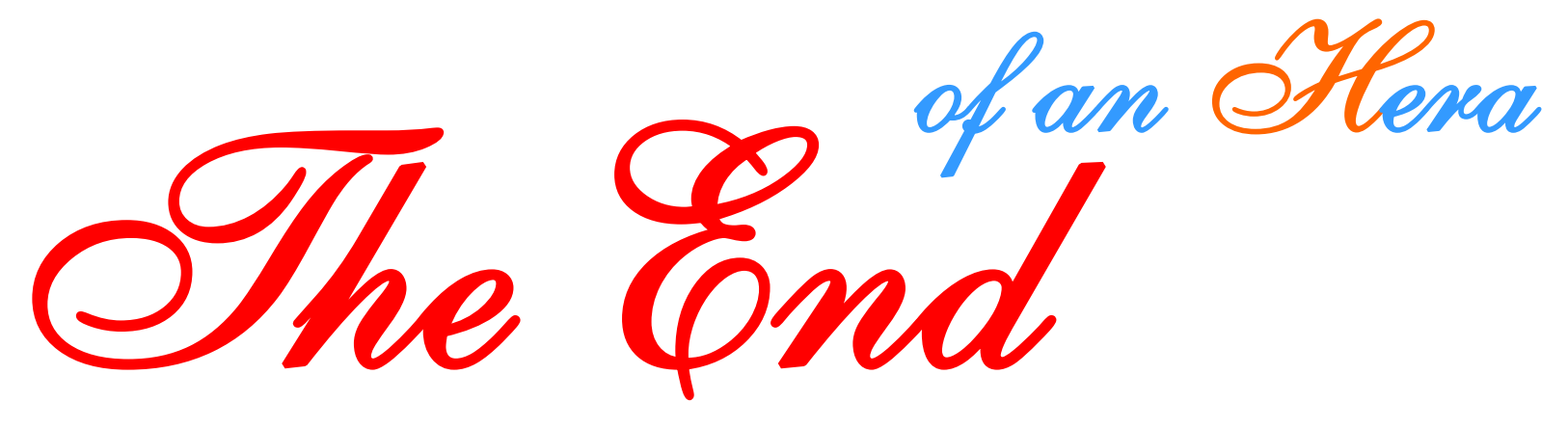




\section{Excited Charm Mesons}

ZEUS:

$$
\begin{aligned}
& D_{1}(2420)^{0}, D_{2}(2460)^{0} \rightarrow D^{*+} \pi_{\mathrm{a}}^{-} \\
& D_{2}(2460) \rightarrow D^{+} \pi_{\mathrm{a}}^{-} \\
& D_{\mathrm{s} 1}{ }^{+}(2536) \rightarrow D^{*+} K_{\mathrm{s}}^{0} \\
& D_{\mathrm{s} 1}{ }^{+}(2536) \rightarrow D^{0} K^{+}
\end{aligned}
$$

measure masses, widths, fragmentation functions, helicity dependence, ..
Spectroscopy of D mesons

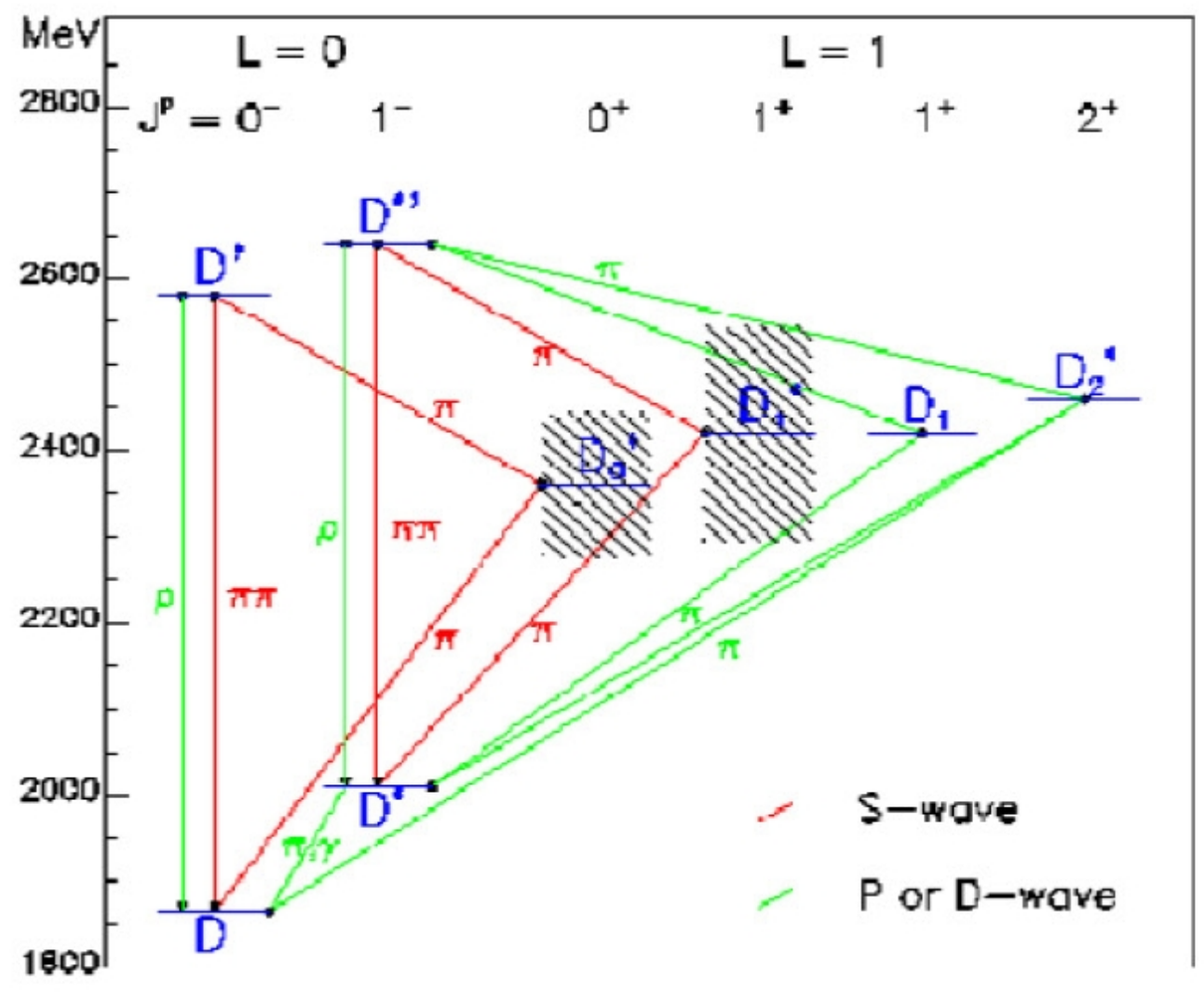




\section{"Backward Analysis"}

Study $D^{*}$ mass in the signal region and in side bands:

Excess is observed only for the signal region with HERA I: the sample is charm enriched.
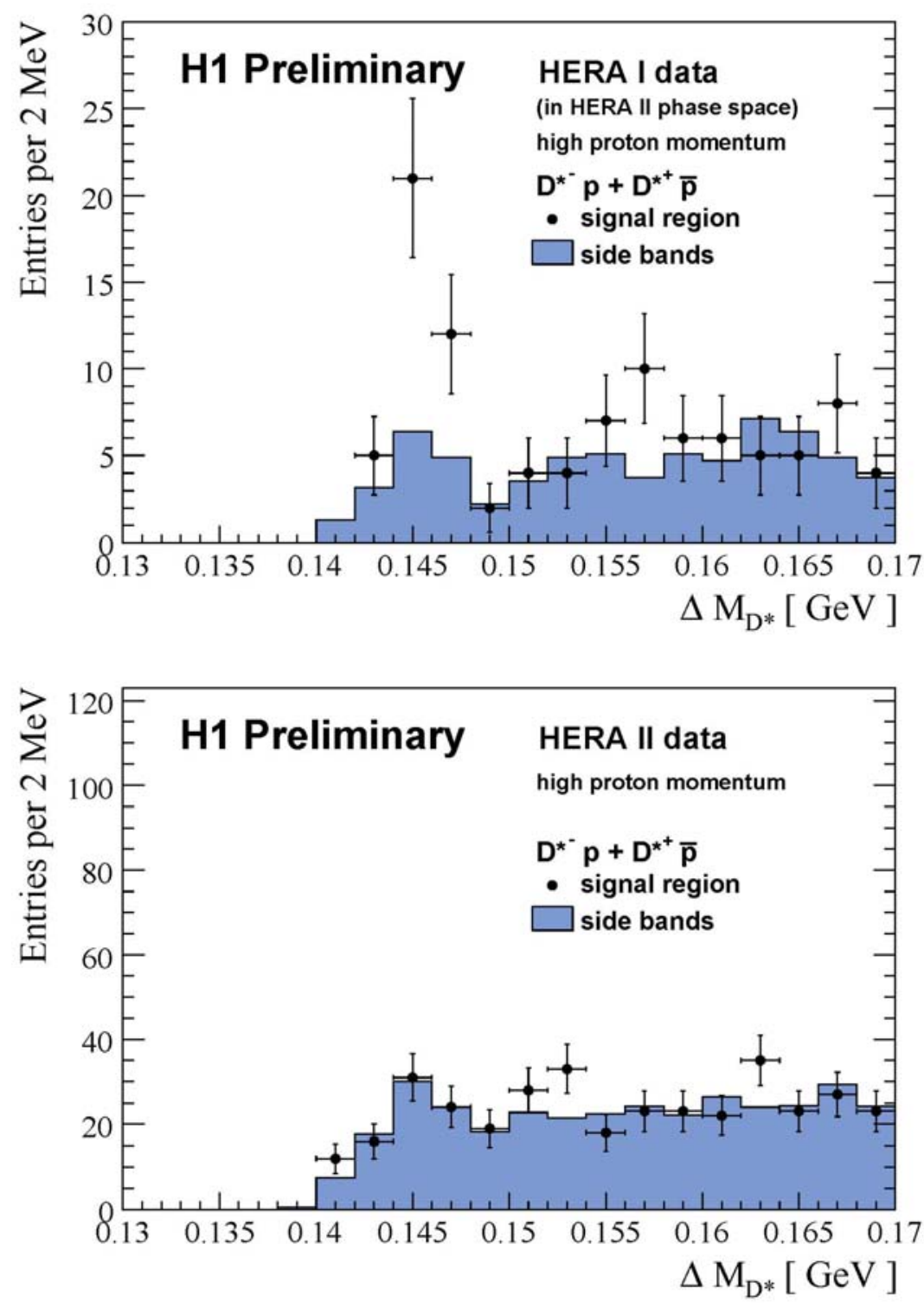